\title{
A Theoretical Comparison of Two Linear Dimensionality Reduction Techniques
}

\author{
Luis Rueda $^{1}$ and Myriam Herrera ${ }^{2}$ \\ ${ }^{1}$ Department of Computer Science and Center for Biotechnology \\ University of Concepción \\ Edmundo Larenas 215, Concepción, Chile \\ Irueda@inf.udec.cl \\ ${ }^{2}$ Department and Institute of Informatics \\ National University of San Juan \\ Cereceto y Meglioli, San Juan, 5400, Argentina \\ mherrera@iinfo.unsj.edu.ar
}

\begin{abstract}
A theoretical analysis for comparing two linear dimensionality reduction (LDR) techniques, namely Fisher's discriminant (FD) and Loog-Duin (LD) dimensionality reduciton, is presented. The necessary and sufficient conditions for which FD and LD provide the same linear transformation are discussed and proved. To derive these conditions, it is first shown that the two criteria preserve the same maximum value after a diagonalization process is applied, and then the necessary and sufficient conditions for various cases, including coincident covariance matrices, coincident prior probabilities, and for when one of the covariances is the identity matrix. A measure for comparing the two criteria is derived from the necessary and sufficient conditions, and used to empirically show that the conditions are statistically related to the classification error for a post-processing quadratic classifier and the Chernoff distance in the transformed space.
\end{abstract}

\section{Introduction}

Linear classifiers have been widely used in pattern recognition due to their simplicity and processing speed. Various schemes that lead to linear classification have been proposed, including the well known Fisher's discriminant (FD) approach [5 20], the perceptron algorithm (the basis of the back propagation neural network learning algorithms) [7 11]14 15], piecewise recognition models [12], random search optimization [13], removal classification structures [1], adaptive linear dimensionality reduction [10] (which outperforms Fisher's classifier for some data sets), linear constrained distancebased classifier analysis [4] (an improvement to Fisher's approach designed for hyperspectral image classification), and recursive Fisher's discriminant [3]. Rueda et al. [18 19] have shown that the optimal classifier between two normally distributed classes can be linear even when the covariance matrices are not equal. In [16], a new approach to selecting the best hyperplane classifier (BHC), which is obtained from the optimal pairwise linear classifier, has been introduced.

A generalization of linear classification can be seen as the process of linear dimensionality reduction (LDR), which aims to reduce high-dimensional data to a lower dimension in such a way that the classification of the new data is more tractable, and 
can still be done efficiently. In this paper, we consider the traditional two-class case, and assume that we are dealing with two classes, $\omega_{1}$ and $\omega_{2}$, which are represented by two normally distributed $n$-dimensional random vectors, $\mathbf{x}_{1} \sim N\left(\mathbf{m}_{1}, \mathbf{S}_{1}\right)$ and $\mathbf{x}_{2} \sim N\left(\mathbf{m}_{2}, \mathbf{S}_{2}\right)$, and whose a priori probabilities are $p_{1}$ and $p_{2}$ respectively. The aim is to linearly transform $\mathbf{x}_{1}$ and $\mathbf{x}_{2}$ into new normally distributed random vectors $\mathbf{y}_{1}$ and $\mathbf{y}_{2}$ of dimension $d, d<n$, using a matrix $\mathbf{A}$ of order $d \times n$, in such a way that the classification error in the transformed space is as small as possible.

A typical approach to reduce the dimension of the data is principal component analysis (PCA) [520 21], but it better applies to unsupervised learning problems, since it takes the whole data as a "single" class, losing the discriminability power of labeled data. We consider two well-known LDR techniques, Fisher's discriminant (FD), and Loog-Duin (LD) dimensionality reduction, and theoretically analyze their common aspects. Let $\mathbf{S}_{W}=p_{1} \mathbf{S}_{1}+p_{2} \mathbf{S}_{2}$ and $\mathbf{S}_{E}=\left(\mathbf{m}_{1}-\mathbf{m}_{2}\right)\left(\mathbf{m}_{1}-\mathbf{m}_{2}\right)^{t}$ be the within-class and between-class scatter matrices respectively. The FD criterion consists of maximizing the distance between the transformed distributions by finding $\mathbf{A}$ that maximizes the following function [5]:

$$
J_{F D}(\mathbf{A})=\operatorname{tr}\left\{\left(\mathbf{A} \mathbf{S}_{W} \mathbf{A}^{t}\right)^{-1}\left(\mathbf{A} \mathbf{S}_{E} \mathbf{A}^{t}\right)\right\} .
$$

The matrix A that maximizes (1) is obtained by finding the eigenvalue decomposition of:

$$
\mathbf{S}_{F D}=\mathbf{S}_{W}^{-1} \mathbf{S}_{E},
$$

and taking the $d$ eigenvectors whose eigenvalues are the largest ones. Since the eigenvalue decomposition of the matrix (2) leads to only one non-zero eigenvalue, $\left(\mathbf{m}_{1}-\right.$ $\left.\mathbf{m}_{2}\right)^{t}\left(\mathbf{m}_{1}-\mathbf{m}_{2}\right)$, whose eigenvector is given by $\left(\mathbf{m}_{1}-\mathbf{m}_{2}\right)$, we can only reduce to dimension $d=1$.

Loog and Duin have recently proposed a new LDR technique for normally distributed classes [8], namely LD, which takes the Chernoff distance in the original space into consideration to minimize the error rate in the transformed space. They consider the concept of directed distance matrices, and a linear transformation in the original space, to finally generalize Fisher's criterion in the transformed space by substituting the within-class scatter matrix for the corresponding directed distance matrix. The LD criterion consists of obtaining the matrix $\mathbf{A}$ that maximizes the function [9]:

$$
\begin{aligned}
J_{L D}(\mathbf{A}) & =\operatorname{tr}\left\{\left(\mathbf{A} \mathbf{S}_{W} \mathbf{A}^{t}\right)^{-1}\right. \\
& {\left.\left[\mathbf{A} \mathbf{S}_{E} \mathbf{A}^{t}-\mathbf{A} \mathbf{S}_{W}^{\frac{1}{2}} \frac{p_{1} \log \left(\mathbf{S}_{W}^{-\frac{1}{2}} \mathbf{S}_{1} \mathbf{S}_{W}^{-\frac{1}{2}}\right)+p_{2} \log \left(\mathbf{S}_{W}^{-\frac{1}{2}} \mathbf{S}_{2} \mathbf{S}_{W}^{-\frac{1}{2}}\right)}{p_{1} p_{2}} \mathbf{S}_{W}^{\frac{1}{2}} \mathbf{A}^{t}\right]\right\} }
\end{aligned}
$$

The solution to this criterion is given by the matrix $\mathbf{A}$ that is composed of the $d$ eigenvectors (whose eigenvalues are maximum) of the following matrix:

$$
\mathbf{S}_{L D}=\mathbf{S}_{W}^{-1}\left[\mathbf{S}_{E}-\mathbf{S}_{W}^{\frac{1}{2}} \frac{p_{1} \log \left(\mathbf{S}_{W}^{-\frac{1}{2}} \mathbf{S}_{1} \mathbf{S}_{W}^{-\frac{1}{2}}\right)+p_{2} \log \left(\mathbf{S}_{W}^{-\frac{1}{2}} \mathbf{S}_{2} \mathbf{S}_{W}^{-\frac{1}{2}}\right)}{p_{1} p_{2}} \mathbf{S}_{W}^{\frac{1}{2}}\right]
$$


In [2], it has been empirically shown that LD outperforms FD in many cases when coupling the LDR technique with a quadratic (Bayesian) classifier in the one dimensional space, namely when the optimal classifier under the assumption of normally distributed classes. As opposed to this, for the linear classifier, FD performs better than LD. In this paper, we derive the necessary and sufficient conditions for which both criteria FD and LD yield the same linear transformation. We also show empirically that the theoretical analysis is related to the probability of error obtained by coupling the LDR technique with quadratic and linear classifiers in the one-dimensional space.

\section{Theoretical Comparison}

Prior to deriving the necessary and sufficient conditions for which FD and LD produce the same linear dimensionality reduction transformation, we show that the maximum for both criteria can be preserved after a simultaneous diagonalization process is applied. We see afterwards that diagonalization allows to simplify the derivations and understanding the conditions. The following lemma shows that if simultaneous diagonalization is applied, the maximum for the FD criterion is preserved.

Lemma 1. Let $\mathbf{x}_{1} \sim N\left(\mathbf{m}_{1}, \mathbf{S}_{1}\right)$ and $\mathbf{x}_{2} \sim N\left(\mathbf{m}_{2}, \mathbf{S}_{2}\right)$ be two normally distributed $n$-dimensional random vectors whose a priori probabilities are $p_{1}$ and $p_{2}$ respectively, where $\mathbf{S}_{\mathbf{1}}$ and $\mathbf{S}_{\mathbf{2}}$ are two arbitrary positive definite and symmetric matrices. Then, there exist two diagonal positive definite matrices $\mathbf{D}_{1}=\mathbf{I}$ and $\mathbf{D}_{2}$, and an $n$-dimensional vector $\boldsymbol{\nu}$, such that $\max _{\{\mathbf{A}\}} J_{F}(\mathbf{A})=\max _{\{\mathbf{A}\}} J_{F}^{\prime}(\mathbf{A})$, where $J_{F}(\mathbf{A})$ is given by (1) and

$$
J_{F}^{\prime}(\mathbf{A})=\operatorname{tr}\left\{\left(\mathbf{A D}_{W} \mathbf{A}^{t}\right)^{-1}\left(\mathbf{A} \mathbf{M}_{E} \mathbf{A}^{t}\right)\right\},
$$

with $\mathbf{D}_{W}=p_{1} \mathbf{D}_{1}+p_{2} \mathbf{D}_{2}$ and $\mathbf{M}_{E}=\boldsymbol{\nu} \boldsymbol{\nu}^{t}$.

The proof of this lemma is accomplished by considering the the following linear transformation: $T(\mathbf{x})=\boldsymbol{\Phi}_{2}^{t} \mathbf{S}_{1}^{-\frac{1}{2}}\left(\mathbf{x}-\mathbf{m}_{1}\right)$. Then, the transformed random vectors have the following parameters: $N(\mathbf{0} ; \mathbf{I})$ y $N\left(\boldsymbol{\nu} ; \mathbf{D}_{2}\right)$, where $\boldsymbol{\nu}=\mathbf{m}_{1}-\mathbf{m}_{2}$. Defining $\mathbf{D}_{W}=$ $p_{1} \mathbf{I}+p_{2} \mathbf{D}_{2}$ and $\mathbf{M}_{E}=\boldsymbol{\nu} \boldsymbol{\nu}^{t}$, it follows that $\mathbf{D}_{W}=\left(\boldsymbol{\Phi}_{2}^{t} \mathbf{S}_{1}^{-\frac{1}{2}}\right) \mathbf{S}_{W}\left(\boldsymbol{\Phi}_{2}^{t} S_{1}^{-\frac{1}{2}}\right)^{t}$ and $\mathbf{M}_{E}=$ $\left(\boldsymbol{\Phi}_{2}^{t} \mathbf{S}_{1}^{-\frac{1}{2}}\right) \mathbf{S}_{E}\left(\boldsymbol{\Phi}_{2}^{t} \mathbf{S}_{1}^{-\frac{1}{2}}\right)^{t}$, which are used to prove that $J_{F}^{\prime}(\mathbf{A})=J_{F}\left(\mathbf{A}\left(\boldsymbol{\Phi}_{2}^{t} \mathbf{S}_{1}^{-\frac{1}{2}}\right)\right)$, and so the maximum values are the same. The complete proof can be found in the unabridged version of this paper [17].

In the following two axioms we prove that the maximum for the LD criterion is the same for any parameters of the normal distribution, and for the corresponding parameters after diagonalization. Although the maximum for both cases coincide, we do not provide the linear transformation that relates the transformation matrix in both cases, before and after diagonalization. This is quite intricate and remains an open problem that we are currently investigating.

Lemma 2. Let $\mathbf{x}_{1} \sim N\left(\mathbf{m}_{1}, \mathbf{S}_{1}\right)$ and $\mathbf{x}_{2} \sim N\left(\mathbf{m}_{2}, \mathbf{S}_{2}\right)$ be two normally distributed $n$-dimensional random vectors whose a priori probabilities are $p_{1}$ and $p_{2}$ respectively, where $\mathbf{S}_{\mathbf{1}}$ and $\mathbf{S}_{\mathbf{2}}$ are two arbitrary positive definite and symmetric matrices. Then, there 
exist two diagonal positive definite matrices $\mathbf{D}_{1}=\mathbf{I}$ and $\mathbf{D}_{2}$, and an $n$-dimensional vector $\boldsymbol{\nu}$, such that $\operatorname{tr}\left\{\mathbf{S}_{L D}\right\}=\operatorname{tr}\left\{\mathbf{D}_{L D}\right\}$, where

$$
\mathbf{D}_{L D}=\mathbf{D}_{W}^{-1}\left[\mathbf{M}_{E}-\mathbf{D}_{W}^{\frac{1}{2}} \frac{p_{1} \log \left(\mathbf{D}_{W}^{-\frac{1}{2}} \mathbf{D}_{1} \mathbf{D}_{W}^{-\frac{1}{2}}\right)+p_{2} \log \left(\mathbf{D}_{W}^{-\frac{1}{2}} \mathbf{D}_{2} \mathbf{D}_{W}^{-\frac{1}{2}}\right)}{p_{1} p_{2}} \mathbf{D}_{W}^{\frac{1}{2}}\right]
$$

with $\mathbf{D}_{W}=p_{1} \mathbf{D}_{1}+p_{2} \mathbf{D}_{2} y \mathbf{M}_{E}=\boldsymbol{\nu} \boldsymbol{\nu}^{t}$.

The proof of this lemma follows by observing that the eigenvalues of a matrix of the form $\mathbf{B}^{-\frac{1}{2}} \mathbf{C B}^{-\frac{1}{2}}$, where $\mathbf{B}$ is a non-singular matrix, are the roots of the polynomial $|\mathbf{C}-\lambda \mathbf{B}|$. In this way, it follows that the eigenvalues of $\mathbf{D}_{w}^{-\frac{1}{2}} \mathbf{D}_{1} \mathbf{D}_{w}^{-\frac{1}{2}}$ coincide with those of $\mathbf{S}_{w}^{-\frac{1}{2}} \mathbf{S}_{1} \mathbf{S}_{w}^{-\frac{1}{2}}$. Also, it is easy to see that the eigenvalues of $\mathbf{D}_{W}^{-\frac{1}{2}} \mathbf{D}_{2} \mathbf{D}_{W}^{-\frac{1}{2}}$ and $\mathbf{S}_{W}^{-\frac{1}{2}} \mathbf{S}_{2} \mathbf{S}_{W}^{-\frac{1}{2}}$ coincide. The proof is accomplished by proving that $\operatorname{tr}\left\{\mathbf{S}_{L D}\right\}=\operatorname{tr}\left\{\mathbf{D}_{L D}\right\}$, after showing that $\operatorname{tr}\{\mathbf{S}\}=\operatorname{tr}\{\mathbf{D}\}$, where:

$$
\mathbf{D}=\frac{p_{1} \log \left(\mathbf{D}_{W}^{-\frac{1}{2}} \mathbf{D}_{1} \mathbf{D}_{W}^{-\frac{1}{2}}\right)+p_{2} \log \left(\mathbf{D}_{W}^{-\frac{1}{2}} \mathbf{D}_{2} \mathbf{D}_{W}^{-\frac{1}{2}}\right)}{p_{1} p_{2}}
$$

and

$$
\mathbf{S}=\frac{p_{1} \log \left(\mathbf{S}_{W}^{-\frac{1}{2}} \mathbf{S}_{1} \mathbf{S}_{W}^{-\frac{1}{2}}\right)+p_{2} \log \left(\mathbf{S}_{W}^{-\frac{1}{2}} \mathbf{S}_{2} \mathbf{S}_{W}^{-\frac{1}{2}}\right)}{p_{1} p_{2}}
$$

The complete proof can be found in [17].

Conjecture 1. Let $\mathbf{x}_{1} \sim N\left(\mathbf{m}_{1}, \mathbf{S}_{1}\right)$ and $\mathbf{x}_{2} \sim N\left(\mathbf{m}_{2}, \mathbf{S}_{2}\right)$ be two normally distributed $n$-dimensional random vectors whose a priori probabilities are $p_{1}$ and $p_{2}$ respectively, where $\mathbf{S}_{\mathbf{1}}$ and $\mathbf{S}_{\mathbf{2}}$ are two arbitrary positive definite and symmetric matrices. Then, there exist two diagonal positive definite matrices $\mathbf{D}_{1}=\mathbf{I}$ and $\mathbf{D}_{2}$, and an $n$-dimensional vector $\boldsymbol{\nu}$, such that $\max _{\{\mathbf{A}\}} J_{L D}(\mathbf{A})=\max _{\{\mathbf{A}\}} J_{L D}^{\prime}(\mathbf{A})$, where $J_{L D}(\mathbf{A})$ is given by (3) and

$$
\begin{aligned}
& J_{L D}^{\prime}(\mathbf{A})=\operatorname{tr}\left\{\left(\mathbf{A} \mathbf{D}_{W} \mathbf{A}^{t}\right)^{-1}\right. \\
& \left.\quad\left[\mathbf{A} \mathbf{M}_{E} \mathbf{A}^{t}-\mathbf{A D}_{W}^{\frac{1}{2}} \frac{p_{1} \log \left(\mathbf{D}_{W}^{-\frac{1}{2}} \mathbf{D}_{1} \mathbf{D}_{W}^{-\frac{1}{2}}\right)+p_{2} \log \left(\mathbf{D}_{W}^{-\frac{1}{2}} \mathbf{D}_{2} \mathbf{D}_{W}^{-\frac{1}{2}}\right)}{p_{1} p_{2}} \mathbf{D}_{W}^{\frac{1}{2}} \mathbf{A}^{t}\right]\right\}
\end{aligned}
$$

with $\mathbf{D}_{W}=p_{1} \mathbf{D}_{1}+p_{2} \mathbf{D}_{2} y \mathbf{M}_{E}=\boldsymbol{\nu} \boldsymbol{\nu}^{t}$.

Proving this result for $d=n$, follows by using Lemma 2 , which states that $\operatorname{tr}\left\{\mathbf{S}_{L D}\right\}=$ $\operatorname{tr}\left\{\mathbf{D}_{L D}\right\}$. It can be shown that $\left.\operatorname{tr}\left\{\left(\mathbf{A D}_{W} \mathbf{A}^{t}\right)^{-1} \mathbf{A}\left[\mathbf{M}_{E}-\cdots\right) \mathbf{A}^{t}\right]\right\}=\operatorname{tr}\left\{\mathbf{D}_{L D}\right\}$, and hence using the same reasoning, this can be shown for $\operatorname{tr}\left\{\mathbf{S}_{L D}\right\}$. For $d<n$, although $\operatorname{tr}\left\{\mathbf{S}_{L D}\right\}=\operatorname{tr}\left\{\mathbf{D}_{L D}\right\}$, it does not easily follow that the $d(d<n)$ eigenvalues of $\mathbf{S}_{L D}$ and $\mathbf{D}_{L D}$ are the same. This has been verified numerically $\sqrt{1}$, but formally proving it remains an open problem. The complete sketch of proof can be found in [17].

\footnotetext{
${ }^{1}$ Proving this formally is not trivial, since one needs to find a linear transformation of the matrix A from the original to the diagonalized space. However the underlying expressions contain non-linear functions such as, $\log$ and powers of $\frac{1}{2}$.
} 
Note that even if we would have shown that the eigenvalues of $\mathbf{D}_{W}$ and $\mathbf{S}_{W}$ are the same, this does not imply that the eigenvectors are coincident too. This can be easily seen as follows. Let $\mathbf{D}$ be any diagonal, non-singular matrix of order $n \times n$, and $\mathbf{S}=\mathbf{F}^{-1} \mathbf{D F}$ any non-singular matrix of order $n \times n$, where $\mathbf{F} \neq \mathbf{I}$ is an arbitrary non-singular matrix of order $n \times n$. Clearly, the eigenvalues of $\mathbf{S}$ can be obtained by means of the decomposition $\mathbf{F S F}^{-1}$, resulting in $\mathbf{D}$. Then, the eigenvalues of $\mathbf{S}$ and $\mathbf{D}$ are the same; however, the corresponding eigenvectors are given by $\mathbf{F}$ and $\mathbf{I}$. For this reason, showing that the eigenvectors of $\mathbf{S}_{L D}$ and $\mathbf{D}_{L D}$ (whose eigenvalues are the largest) are given by $\mathbf{A}$ and $\mathbf{A C}$ respectively, where $\mathbf{C}$ is an arbitrary transformation matrix, is an interesting problem in the sense that it would allow to find the solution in the "diagonalized" space, allowing to transform the matrix A back to the "original" space. This is a problem that we are currently investigating.

We now formalize the necessary and sufficient conditions for which the two LDR methods we study, FD and LD, produce the same linear transformation.

Theorem 1. Let $\mathbf{x}_{1} \sim N\left(\mathbf{m}_{1}, \mathbf{S}_{1}\right)$ and $\mathbf{x}_{2} \sim N\left(\mathbf{m}_{2}, \mathbf{S}_{2}\right)$ be two normally distributed $n$-dimensional random vectors whose a priori probabilities are $p_{1}$ and $p_{2}$ respectively, where $\mathbf{S}_{1}$ and $\mathbf{S}_{\mathbf{2}}$ are two diagonal positive definite and symmetric matrices. Then, $J_{F}(A)=J_{L D}(A)$ if and only if:

$$
\mathbf{S}_{1}^{p_{1}} \mathbf{S}_{2}^{p_{2}}=p_{1} \mathbf{S}_{1}+p_{2} \mathbf{S}_{2}
$$

The proof of this theorem is accomplished by finding the conditions for which $J_{F}(\mathbf{A})=$ $J_{L D}(\mathbf{A})$ holds if and only if:

$$
\mathbf{S}_{W}^{\frac{1}{2}} \frac{p_{1} \log \left(\mathbf{S}_{W}^{-\frac{1}{2}} \mathbf{S}_{1} \mathbf{S}_{W}^{-\frac{1}{2}}\right)+p_{2} \log \left(\mathbf{S}_{W}^{-\frac{1}{2}} \mathbf{S}_{2} \mathbf{S}_{W}^{-\frac{1}{2}}\right)}{p_{1} p_{2}} \mathbf{S}_{W}^{\frac{1}{2}}=\mathbf{0},
$$

where $\mathbf{0}$ is a zero matrix of order $n \times n$. Using Lemma 1 and Conjecture 1 , it is always possible to obtain the same result for both criteria). We ensure, in this way, that $\mathbf{S}_{1}=$ $\operatorname{diag}\left(\lambda_{1_{i}}\right)$ and $\mathbf{S}_{2}=\operatorname{diag}\left(\lambda_{2_{i}}\right)$. For the two LDR criteria to coincide the following must hold:

$$
\forall i: \lambda_{1_{i}}^{p_{1}} \lambda_{2_{i}}^{p_{2}}=p_{1} \lambda_{1_{i}}+p_{2} \lambda_{2_{i}}
$$

which can be written in a matrix-form-like manner as in 10 . The complete proof of this theorem can be found in [17]. The following results show the necessary and sufficient conditions for various cases, including coincident covariance matrices, coincident prior probabilities, and for when one of the covariances is the identity matrix. The complete proofs of the corollaries can be found in [17].

Corollary 1. Under the conditions of Theorem 1 if $\mathbf{S}_{1}=\mathbf{S}_{2}$, then $J_{F}=J_{L D}$ for any values of $p_{1}$ and $p_{2}$.

Corollary 2. Under the conditions of Theorem 1 if $\mathbf{S}_{1} \neq \mathbf{S}_{2}$, and (10) holds, then $p_{1} \neq p_{2}$. 
Corollary 3. Under the conditions of Theorem $\square$ if $\mathbf{S}_{1}=\mathbf{I}$ and $\mathbf{S}_{2}=\operatorname{diag}\left(\lambda_{i}\right)$, $J_{F}(\mathbf{A})=J_{L D}(\mathbf{A})$ if and only if:

$$
\lambda_{i}^{p_{2}}-p_{2} \lambda_{i}-p_{1}=0 .
$$

Note that the formulas we found, although are the necessary and sufficient conditions for the coincidence of both criteria, can also be used to evaluate the "similarity" between the classifiers used after the transformation. Also, this kind of analysis can be done using the parameters of the distributions without transforming the data onto the new "diagonalized" space, as it is required by Lemma 1 and Conjecture 1. As a measure for this similarity, and assuming that the underlying covariance matrices are given by I and $\boldsymbol{\Lambda}=\operatorname{diag}\left(\lambda_{1}, \ldots, \lambda_{n}\right)$ respectively, we can use the condition given in (13), and average over all the $n$ dimensions as follows:

$$
\begin{aligned}
\delta & =\frac{1}{n} \sum_{i=1}^{n}\left|\lambda_{i}^{p_{2}}-p_{2} \lambda_{i}-p_{1}\right| \\
& =\left|\frac{1}{n}\left[\operatorname{tr}\left\{\boldsymbol{\Lambda}^{p_{2}}\right\}-p_{2} \operatorname{tr}\{\boldsymbol{\Lambda}\}\right]-p_{1}\right| .
\end{aligned}
$$

In the following section, we utilize this measure to empirically compare the result of the two LDR techniques when coupled with either of two classifiers, linear or quadratic.

\section{Empirical Results}

The tests involve ten different datasets of dimensions $n=10,20, \ldots, 100$ each with two randomly generated normally distributed classes. The underlying parameters of the distributions were generated as follows. Each element of the means, $\mathbf{m}_{1}$ and $\mathbf{m}_{2}$, was generated by following distributions $\mathrm{U}[0, b / n]$ and $\mathrm{U}[b / n, 2 b / n]$, where $b$ was set to 10. Dividing by $n$ makes sure that the classification task is not easier when increasing the dimension. The eigenvalues of the covariances, $\mathbf{S}_{1}$ and $\mathbf{S}_{2}$, were randomly generated as $\mathrm{U}[0, b]$, and the corresponding eigenvectors from a random matrix in $\mathrm{U}(0, b / n)$ followed by a $\mathrm{QR}$ decomposition, taking the orthogonal matrix $\mathbf{Q}$. This ensures that the covariances are positive and definite. A linear transformation using $\mathbf{S}_{1}^{-\frac{1}{2}}$ was applied, obtaining covariances $\mathbf{I}$ and $\mathbf{S}_{1}^{-\frac{1}{2}} \mathbf{S}_{2} \mathbf{S}_{1}^{-\frac{1}{2}}$ respectively, followed by a subsequent linear transformation using $\boldsymbol{\Phi}_{2}$, which contains the eigenvectors of $\mathbf{S}_{1}^{-\frac{1}{2}} \mathbf{S}_{2} \mathbf{S}_{1}^{-\frac{1}{2}}$. After all the transformations, the underlying covariance matrices resulted in $\mathbf{I}$ and $\boldsymbol{\Lambda}_{2}$. We also randomly generated $p_{1}$ as a $\mathrm{U}[0.3,0.7]$ and assigned $p_{2}=1-p_{1}$. We trained two LDR techniques, FD and LD, using these parameters, and for each dataset we generated 10,000 samples for testing purposes. After a linear transformation to dimension $d=1$ is performed we have tested two classifiers: the linear $(\mathrm{L})$ classifier, which is obtained by averaging the covariances matrices in the transformed space, and the quadratic $(\mathrm{Q})$ classifier which is the one that minimizes the error rate assuming that the parameters in the transformed data are given by $\mathbf{A m}_{i}$ and $\mathbf{A} \mathbf{S}_{i} \mathbf{A}^{t}$. 
The classification error for FD and LD and the corresponding values of $\delta$ are shown in Table 1 . The second and third columns contain the classification error for FD and LD coupled with a quadratic (Q) classifier, and the fourth column the difference (in absolute value) between the two errors. The fifth, sixth and seventh columns represent the same but for the linear (L) classifier, and the last column contains the value of $\delta$, which is computed as in (15). For the quadratic classifier, the smallest value of $\delta$ coincides with the smallest difference, and the third difference corresponds to the fourth value of $\delta$. Note that the four largest values of $\delta$ correspond to values which are among the five largest difference of errors. For the linear classifier, we note that the four largest values of $\delta$ correspond to the differences which are among the fifth largest ones; however not all small values of $\delta$ correspond to the smallest error differences. To numerically assess this correspondence, we computed the Spearman rank correlation coefficient for two pairs of lists [6], the differences for the quadratic classifier and $\delta$, and the differences for the linear classifier and $\delta$, respectively. The coefficients obtained are 0.62 for the quadratic classifier, and 0.32 for the linear classifier. This denotes that in both cases, the values of $\delta$ and the differences are positively correlated. The high value for the quadratic classifier indicates that the latter is quite related with the differences between the eigenvalues of the underlying covariance matrices for the optimal classifier in the transformed space. This is not the case for the linear classifier.

Table 1. Error rates and differences for two LDR techniques, FD and LD, coupled with quadratic and linear classifiers

\begin{tabular}{|r|c|c|c|c|c|c|c|}
\hline$d$ & $\mathrm{FD}(\mathrm{Q})$ & $\mathrm{LD}(\mathrm{Q})$ & Diff. & $\mathrm{FD}(\mathrm{L})$ & $\mathrm{LD}(\mathrm{L})$ & Diff. & $\delta$ \\
\hline 10 & 0.2629 & 0.2460 & 0.0169 & 0.2639 & 0.3404 & 0.0765 & 0.1280 \\
\hline 20 & 0.2004 & 0.1132 & 0.0872 & 0.2012 & 0.2083 & 0.0071 & 0.4572 \\
\hline 30 & 0.2865 & 0.1803 & 0.1062 & 0.2870 & 0.4296 & 0.1426 & 0.4801 \\
\hline 40 & 0.2747 & 0.2179 & 0.0568 & 0.3053 & 0.3524 & 0.0471 & 0.4690 \\
\hline 50 & 0.2646 & 0.1262 & 0.1384 & 0.2659 & 0.2858 & 0.0199 & 0.2712 \\
\hline 60 & 0.2190 & 0.0778 & 0.1412 & 0.2216 & 0.3646 & 0.1430 & 0.6132 \\
\hline 70 & 0.2026 & 0.0407 & 0.1619 & 0.2281 & 0.3809 & 0.1528 & 0.6919 \\
\hline 80 & 0.3313 & 0.0989 & 0.2324 & 0.3318 & 0.3500 & 0.0182 & 0.6702 \\
\hline 90 & 0.3859 & 0.0835 & 0.3024 & 0.3920 & 0.2968 & 0.0952 & 0.9946 \\
\hline 100 & 0.3191 & 0.1433 & 0.1758 & 0.3195 & 0.3417 & 0.0222 & 0.2308 \\
\hline
\end{tabular}

To analyze the relation between $\delta$ and the quadratic classifier, we computed the Chernoff distance between both distributions in the transformed space as follows

$$
\begin{aligned}
k(\beta, \mathbf{A})= & \frac{\beta(1-\beta)}{2}\left(\mathbf{A} \mathbf{m}_{\mathbf{1}}-\mathbf{A} \mathbf{m}_{\mathbf{2}}\right)^{t}\left[\beta \mathbf{A} \mathbf{S}_{1} \mathbf{A}+(1-\beta) \mathbf{A} \mathbf{S}_{2} \mathbf{A}\right]^{-1}\left(\mathbf{A} \mathbf{m}_{\mathbf{1}}-\mathbf{A} \mathbf{m}_{\mathbf{2}}\right) \\
& +\frac{1}{2} \log \frac{\left|\beta \mathbf{A} \mathbf{S}_{1} \mathbf{A}+(1-\beta) \mathbf{A} \mathbf{S}_{2} \mathbf{A}\right|}{\left|\mathbf{A} \mathbf{S}_{1} \mathbf{A}\right|^{\beta}\left|\mathbf{A} \mathbf{S}_{2} \mathbf{A}\right|^{1-\beta}} .
\end{aligned}
$$

where $\beta=p_{1}$ and $\mathbf{A}$ is the linear transformation matrix obtained by either FD or $\mathrm{LD}$, and compared it with the corresponding values of $\delta$. The results are tabulated in 
Table 2. The Chernoff distances in the transformed space for FD and LD are given in the second and third columns, while their difference in absolute value is given in the fourth column. We observe that, as in the error for the quadratic classifier, the four largest values of $\delta$ correspond to those differences which are among the five largest ones. A similar situation occurs with the smallest values of $\delta$. Again, to numerically assess the relation between the difference ("Diff." in the table) and $\delta$, we computed the Spearman rank correlation coefficient, resulting in 0.65 , which is a significantly large value, and indicates a high positive correlation between the two lists.

Table 2. Chernoff distance in the transformed space after the two LDR transformations, FD and LD, are applied

\begin{tabular}{|r|c|c|c|c|}
\hline$d$ & FD & LD & Diff. & $\delta$ \\
\hline 10 & 1.5286 & 2.2260 & 4.2803 & 0.1280 \\
\hline 20 & 0.7924 & 4.5833 & 0.6974 & 0.4572 \\
\hline 30 & 1.1314 & 3.5879 & 3.7908 & 0.4801 \\
\hline 40 & 1.1156 & 2.5607 & 2.4565 & 0.4690 \\
\hline 50 & 0.4929 & 5.1266 & 1.4452 & 0.2712 \\
\hline 60 & 2.1272 & 8.5170 & 4.6337 & 0.6132 \\
\hline 70 & 2.6234 & 12.2768 & 6.3899 & 0.6919 \\
\hline 80 & 0.3178 & 6.8664 & 9.6534 & 0.6702 \\
\hline 90 & 0.3454 & 7.3926 & 6.5486 & 0.9946 \\
\hline 100 & 0.2593 & 4.5395 & 7.0472 & 0.2308 \\
\hline
\end{tabular}

\section{Conclusion}

We have formalized the necessary and sufficient conditions for which two well-known LDR techniques, FD and LD, provide the same linear transformation. To derive these conditions, we have first shown that the two criteria preserve the same maximum value after a diagonalization process is applied. For FD, we have found the linear transformation that allows to obtain the LDR in the original space. For the LD criterion, however, we have only shown that the maximum values coincide in both original and diagonalized space, and conjectured that this holds for lower dimensional spaces.

We have derived the necessary and sufficient conditions for various cases, including coincident covariance matrices, coincident prior probabilities, and for when one of the covariances is the identity matrix. We have empirically shown that the conditions are statistically related to the classification error for a post-processing quadratic classifier and the Chernoff distance in the transformed space.

One of the problems that constitute a future extension of this work is to analyze the correspondence between the two LDR methods for more than two classes. Another problem involves deriving the expressions for obtaining the LD dimensionality reduction in the original space, when the diagonalized distributions are given. This is, though quite intricate, an interesting problem as it would allow to speed up the LDR derivation significantly. 
Acknowledgments. This research has been supported by the Chilean National Council for Technological and Scientific Research, FONDECYT grant No. 1060904, and the Institute of Informatics, National University of San Juan.

\section{References}

1. M. Aladjem. Linear Discriminant Analysis for Two Classes Via Removal of Classification Structure. IEEE Trans. on Pattern Analysis and Machine Intelligence, 19(2):187-192, 1997.

2. M. Ali, L. Rueda, and M. Herrera. On the Performance of Chernoff-distance-Based Linear Dimensionality Reduction Techniques. In Proc. of the 18th Canadian Conference on Artificial Intelligence, volume LNAI 4013, pages 469-480. Springer, 2006.

3. T. Cooke. Two Variations on Fisher's Linear Discriminant for Pattern Recognition. IEEE Transations on Pattern Analysis and Machine Intelligence, 24(2):268-273, 2002.

4. Q. Du and C. Chang. A Linear Constrained Distance-based Discriminant Analysis for Hyperspectral Image Classification. Pattern Recognition, 34(2):361-373, 2001.

5. R. Duda, P. Hart, and D. Stork. Pattern Classification. John Wiley and Sons, Inc., New York, NY, 2nd edition, 2000.

6. E. Lehmann and H. D'Abrera. Nonparametrics: Statistical Methods Based on Ranks. Prentice-Hall, 1998.

7. R. Lippman. An Introduction to Computing with Neural Nets. In Neural Networks: Theoretical Foundations and Analsyis, pages 5-24. IEEE Press, 1992.

8. M. Loog and P.W. Duin. Linear Dimensionality Reduction via a Heteroscedastic Extension of LDA: The Chernoff Criterion. IEEE Transactions on Pattern Analysis and Machine Intelligence, 26(6):732-739, 2004.

9. M. Loog and R. Duin. Non-iterative Heteroscedastic Linear Dimension Reduction for TwoClass Data. In Proceedings of the Joint IAPR International Workshops SSPR 2002 and SPR 2002, volume LNCS 2396, pages 508-517. Springer, 2002.

10. R. Lotlikar and R. Kothari. Adaptive Linear Dimensionality Reduction for Classification. Pattern Recognition, 33(2):185-194, 2000.

11. O. Murphy. Nearest Neighbor Pattern Classification Perceptrons. In Neural Networks: Theoretical Foundations and Analysis, pages 263-266. IEEE Press, 1992.

12. A. Rao, D. Miller, K. Rose, , and A. Gersho. A Deterministic Annealing Approach for Parsimonious Design of Piecewise Regression Models. IEEE Transactions on Pattern Analysis and Machine Intelligence, 21(2):159-173, 1999.

13. S. Raudys. On Dimensionality, Sample Size, and Classification Error of Nonparametric Linear Classification. IEEE Transactions on Pattern Analysis and Machine Intelligence, 19(6):667-671, 1997.

14. S. Raudys. Evolution and Generalization of a Single Neurone: I. Single-layer Perception as Seven Statistical Classifiers. Neural Networks, 11(2):283-296, 1998.

15. S. Raudys. Evolution and Generalization of a Single Neurone: II. Complexity of Statistical Classifiers and Sample Size Considerations. Neural Networks, 11(2):297-313, 1998.

16. L. Rueda. Selecting the Best Hyperplane in the Framework of Optimal Pairwise Linear Classifiers. Pattern Recognition Letters, 25(2):49-62, 2004.

17. L. Rueda and M. Herrera. Necessary and Sufficient Conditions for the Equivalence of two Linear Dimensionality Reduction Techniques. Submitted for Publication, 2006. Electronically available at http://www.inf.udec.cl/ lrueda/papers/FisherLoogDuinJnl.pdf. 
18. L. Rueda and B. J. Oommen. On Optimal Pairwise Linear Classifiers for Normal Distributions: The Two-Dimensional Case. IEEE Transations on Pattern Analysis and Machine Intelligence, 24(2):274-280, 2002.

19. L. Rueda and B. J. Oommen. On Optimal Pairwise Linear Classifiers for Normal Distributions: The $d$-Dimensional Case. Pattern Recognition, 36(1):13-23, January 2003.

20. S. Theodoridis and K. Koutroumbas. Pattern Recognition. Elsevier Academic Press, third edition, 2006.

21. A. Webb. Statistical Pattern Recognition. John Wiley \& Sons, N.York, second edition, 2002. 